\title{
Breast core needle biopsy: issues and controversies
}

\author{
Michael Bilous
}

Institute of Clinical Pathology and Medical Research, Westmead Hospital and Sydney University, Sydney, Australia

\begin{abstract}
Core needle biopsy (CNB) is increasingly being used in the investigation of breast disease whether this is asymptomatic and suspected after screening mammography, or presents symptomatically in the patient. In most cases, the result of the procedure provides a definitive diagnosis or at least provides information that is used to plan the further management of the patient. There are, however, a number of unresolved issues with the use of CNB; for example, with regard to the amount of information that can be reliably derived from CNB or with regard to the management of the patient after some CNB diagnoses. Oestrogen and progesterone receptors and HER2 are reported on both core biopsies and excision specimens, but there continues to be debate over which represents the more appropriate specimen type on which to perform these tests. There are a number of possible diagnoses after CNB for which the management is not straightforward and around which there may be controversy, or just a lack of sufficient evidence to support a definite management plan. These 'lesions of uncertain malignant potential' include papillary lesions, fibroepithelial lesions with cellular stroma, mucocoelelike lesions and radial scars. Currently, these are removed surgically but there may be an argument for more conservative management including vacuum-assisted core excision in some cases.

Modern Pathology (2010) 23, S36-S45; doi:10.1038/modpathol.2010.34
\end{abstract}

Keywords: breast; core needle biopsy

Some of the issues regarding the use, interpretation and follow-up of patients having a core needle breast biopsy (CNB) are addressed below under the following headings:

- Role and indications for CNB

- Pathology reporting of CNB

- Diagnostic problems

- When to excise after CNB

- Complications and pathology after CNB

\section{Role and indications for CNB}

The principal aim of CNB is to provide a diagnosis of a breast abnormality before, and in many cases avoiding the need for, open surgical biopsy. As a requirement for participation in the National Mammographic Screening Program in Australia, all breast screening and assessment centres are required

Correspondence: Dr M Bilous, MD, Institute of Clinical Pathology and Medical Research, Westmead Hospital and Sydney University, Institute Road, Sydney 2145, Australia.

E-mail: michael.bilous@swahs.health.nsw.gov.au

Received 7 January 2010; accepted 8 January 2010 to have provided an unequivocal pathology diagnosis of cancer in at least $75 \%$ of patients before they proceed to surgery. CNB has been progressively replacing fine needle aspiration biopsy (FNAB) in this preoperative assessment role and is clearly the method of choice in the investigation of microcalcifications without an associated mass lesion. However, in many centres CNB is still used as an additional investigation tool when FNAB is either not available or has failed to produce a diagnosis.

The choice of which CNB device to use is made by the radiologist, breast physician or surgeon and is determined by a number of interrelated factors with regard to the nature of the breast abnormality (palpable or impalpable), the availability of the various core biopsy instruments (conventional or vacuum-assisted), the imaging modalities (mammography, ultrasound or MRI), the cost and patientspecific factors such as their age and ability to undergo the biopsy procedure. For example, the mammographic finding of indeterminate microcalcifications in a 60-year-old woman, in the absence of a mass lesion or architectural distortion, and not visualized by ultrasound, might best be investigated by stereotactic-guided vacuum-assisted core biopsy 
(VACB) to maximize the amount of tissue available for diagnosis. Alternatively a well-defined, palpable, mass lesion in a 20-year-old woman might be correctly diagnosed using a conventional $14 \mathrm{~g}$ CNB with two or three passes using freehand or ultrasound imaging guidance.

Although a comprehensive knowledge of imaging is not a pre-requisite for pathologists reporting CNB, it helps in the interpretation of the pathology findings if the pathologist appreciates the correlation between imaging categories and pathology. This knowledge is gained largely through regular meetings between the radiologist and pathologist, at which the pathology and imaging results for specific patients are presented, and outcomes and further management are discussed in a multidisciplinary setting. The radiology-pathology correlation of CNB results is greatly assisted by specimen radiology of all cores performed for microcalcifications, the separation of cores containing calcification from those without at the time of the procedure and the availability of the specimen radiograph to the pathologist at the time of reporting. For CNB performed for masses and architectural distortions, the nature of the imaging abnormality should be clearly stated on the pathology request form.

\section{Pathology reporting of CNB}

CNB are processed in a routine manner to paraffin embedding. The protocol for sectioning will vary between laboratories, with some centres examining a minimum of three H\&E-stained sections cut at $50 \mu$ intervals. This should allow preservation of sufficient material in the paraffin block for further levels and immunohistochemistry to be performed. In our laboratory, we cut nine serial sections and H\&E stain levels 1,4 and 7 . The six unstained sections are retained for immunohistochemistry or further H\&E staining as required. Paraffin block radiography may be necessary to identify microcalcifications not seen in the sections, and the separation of cores containing calcium from those without at the time of core specimen radiography is very helpful and should be mandatory.

Possible pitfalls are the loss of microcalcifications at the time of section cutting, especially if these are coarse, calcium that is left in the formalin of the specimen container and failure to recognize calcium oxalate deposition in the CNB.

In the majority of cases a definite diagnosis is performed after CNB; for example, fibroadenoma (FA), invasive carcinoma. For these patients a clear management plan can be determined preferably by a multidisciplinary management team. Other cases will require correlation between the radiology and pathology to establish that the area(s) of interest or microcalcifications targeted by the core biopsy have been seen by the pathologist.
Some specific lesions are known to have a high incidence of 'upgrading' after excision ('underestimation' by CNB), and surgical excision of the whole area is required. Examples are atypical ductal hyperplasia (ADH) upgraded to ductal carcinoma in situ (DCIS), lobular neoplasia (LN) upgraded to invasive carcinoma and flat epithelial atypia (FEA) upgraded to DCIS or invasive carcinoma. Also included in this category are those lesions that may be heterogeneous such as papillary lesions. The sample obtained by CNB may not have included the most significant area. For example, an intraduct papilloma diagnosed on CNB may be found to be an intraduct papillary carcinoma after excision and examination of the entire lesion.

As part of the 'triple test' used in the assessment of screen-detected lesions, a pathology category classification (B1-5) is used by the UK National Health Service Breast Screening Program (NHSBSP) and by other mammographic screening programs including that established in Australia; see abbreviated summary and examples below. ${ }^{1}$ The pathology category given by the pathologist in the report is then used to determine future management of the patient together with the other two components of the 'triple test', ie, the clinical examination and history, and the imaging findings. It is important to note that this classification is not used to replace the pathology description of the findings but to supplement the pathology report and to assist in future management decisions.

B1 (normal tissue): Normal breast or other normal tissue, including adipose tissue, may include microcalcifications associated with atrophic or normal terminal duct lobular units (TDLUs).

B2 (benign lesion): FA, fat necrosis, duct ectasia.

B3 (lesion of uncertain malignant potential): Includes ADH, LN, fibroepithelial lesions with cellular stroma and phyllodes tumours (PTs), papillary lesions, FEA and radial scar.

B4 (suspicious): A definite malignant diagnosis (DCIS or invasive carcinoma) is not possible because of crush artifact, poor fixation or a small questionable focus of non-diagnostic cells.

B5 (malignant): An unequivocal malignant diagnosis (includes DCIS and invasive carcinoma).

The management of the various B3 lesions has been the subject of a number of studies designed to establish which lesions require surgical excision and which can be followed-up without surgery. Currently, a large majority of these lesions is managed by open surgical excision. However, there is a case for looking at alternative options such as vacuum-assisted core excision and follow-up without further tissue examination, in selected cases. See below in the section 'When to excise after CNB'.

\section{CNB Reporting of DCIS}

Microcalcifications detected on mammography were found in $72 \%$ of women with clinically occult DCIS 
in 100 women reported by Stomper et al. ${ }^{2}$ In $10 \%$ a soft tissue abnormality was seen and in $12 \%$ there was a combination of the two. DCIS is therefore a common form of malignancy detected by mammography and investigated by CNB.

The pathology report of DCIS in a CNB should include a description of nuclear grade (low, intermediate or high), architectural type(s) and the presence of necrosis (comedo or punctate type). ${ }^{3}$ The presence of microcalcification should also be documented to correlate with the imaging findings. There is good evidence of concordance between the CNB and excision specimens for these microscopic features which are important in predicting both the risk of recurrence after breast conservation and radiotherapy, and the risk of progression to invasive carcinoma. $^{3,4}$

\section{CNB Reporting of Invasive Carcinoma}

How much information is provided by the pathologist from a CNB showing an invasive carcinoma will vary between pathologists and institutions. It will also depend upon how much pre-operative information is required before surgical therapy is instituted and also on whether the information is equivalent to (and as reliable as) that derived from an excision specimen. There is strong support for the provision of only one set of results for each cancer, and historically this has been derived from the excised specimen. One set of results avoids the possibility of misleading and conflicting findings from specimens tested at different times, possibly at different laboratories and using different specimens.

There is generally good correlation between prognostic factors derived from $\mathrm{CNB}$ and the subsequently excised specimen, ${ }^{5,6}$ although histological grade may be underestimated from the CNB as a result of a lower mitotic rate being seen with the small amount of tissue available for assessment. ${ }^{7}$ If neoadjuvant therapy is planned, then the full range of prognostic and predictive information must be provided from the CNB. This will include histological type, histological grade and the presence of lymphovascular invasion, if definitely identified.

The accuracy of assessment of the predictive factors ER, PR and HER2 in core biopsies when compared with excision specimens is still the subject of debate. Investigation of this issue is complicated by the known interlaboratory and intralaboratory variation in testing procedures and scoring seen in quality assurance programs for all three markers. ${ }^{8,9}$ Wood et $a l^{10}$ found a concordance rate of $95.8 \%$ for ER and $90.3 \%$ for PR between CNB and excision specimens. Interestingly, the authors suggest that the lower rate of PR staining in excision specimens suggested that it was more sensitive than ER to fixation or processing. The concordance rate was lower for HER2 at $86.6 \%$. Immunohistochemistry was used to assess all three markers without
FISH confirmation of the HER2 result. ${ }^{10}$ In this series of 100 patients, there were 4 patients for whom the difference in HER2 staining would have influenced treatment decisions; 1 with a negative core and a positive excision specimen and 3 for which the core biopsy was equivocal and the excision positive. Other series have shown a $100 \%$ concordance for ER and HER2. ${ }^{11}$ By contrast, Mann et $a l^{12}$ showed a discordance rate of $14 \%$ for ER, $17 \%$ for PR and $20 \%$ for HER2. In $54 \%$ of the CNB, the HER2 staining was more intense in the core biopsy and in only $4 \%$ the reverse was true. Several other studies have shown a discordance rate for HER2 results varying from 4 to $9 \% .{ }^{13-16}$

There is evidence that larger tumours may show a higher discordance rate for ER and PR presumably due to heterogeneity in the tumour being missed in the core biopsy ${ }^{17}$ (Figure 1).
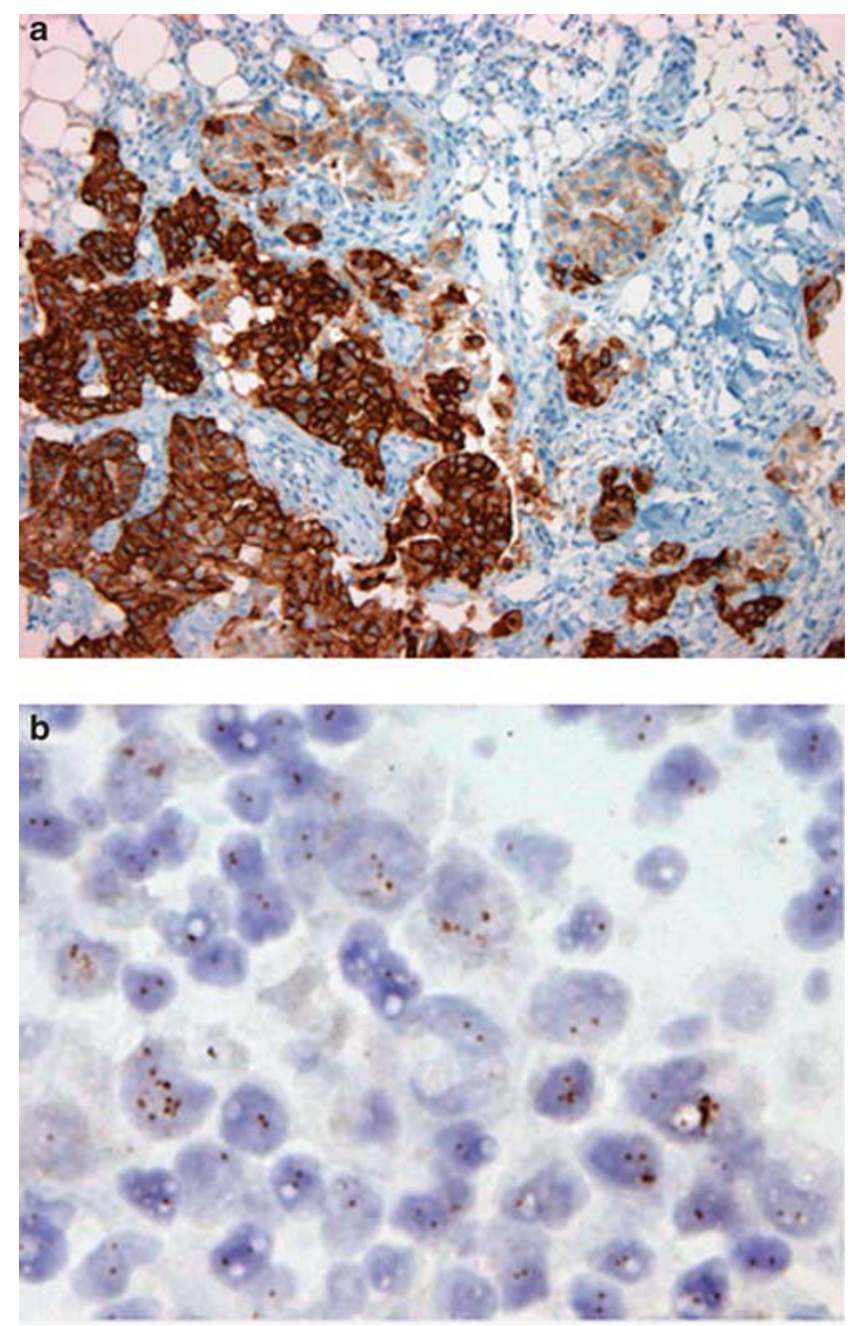

Figure 1 HER2 testing of CNB; two other cores from the same patient were completely negative; (a) HER2 IHC (4B5, Ventana Medical Systems, AZ, USA) shows $3+, 2+$ and $1+$ staining; (b) HER2 ISH (Invitrogen Spot-Light CISH) shows heterogeneity of HER2 gene copy number from 1 and 2 (non-amplified) to 12 (amplified) in the same core. 
The result of HER2 testing has profound treatment and budgetary implications. Most testing methods have been developed using surgical specimens rather than CNB. The recommended fixation, processing and antigen retrieval are suitable for these larger specimens, but are not necessarily optimal for the small CNB specimen. Other issues such as the absence of a negative control (normal ducts) in the CNB could lead to a false-positive result, and a biclonal carcinoma could be missed as a result of sampling.

There is also a lack of data to confirm whether in discordant cases the HER2 result on the core or the excision specimen most accurately reflects treatment response. In some centres, the surgeon or oncologist requests the results of ER, PR and HER2 from the core biopsy before deciding on a treatment plan. However, if this is not the case then the following testing regimen is suggested:

Patients having neoadjuvant therapy: Test the CNB for ER, PR and HER2 and use FISH or brightfield ISH as the HER2 test, if any staining is seen (ie, retest all $1+, 2+$ and $3+$ cases by ISH).

Re-test for ER, PR and HER2 any subsequent specimens received from these patients.

Patients undergoing surgical excision: Test the surgical excision specimen for ER, PR and HER2, and if either ER or PR is negative, test the core biopsy for ER and/or PR to confirm the result.

\section{Diagnostic problems (and when to recommend excision after a CNB)}

CNB is an accurate method for diagnosing breast lesions, correctly identifying benign and malignant disease in more than $90 \%$ of cases. Certain types of lesion, however, present diagnostic difficulty and/or are associated with a more significant lesion. In these cases, the whole area may need to be excised and assessed even after immunohistochemical work-up. Examples include the following:

- Fibroepithelial lesions with cellular stroma and PTs.

- Spindle cell lesions.

- Residual or recurrent carcinoma (in situ or invasive) after radiation therapy.

- Papillary lesions.

- Mucinous lesions.

- Radial scar.

- Atypical proliferative lesions including FEA, ADH and LN.

- Microcalcifications not associated with a specific pathology but suspicious of origin in DCIS.

Some of these will be discussed below.

\section{Fibroepithelial Lesions}

The diagnosis by CNB of a FA at one end of the spectrum and a malignant PT at the other is usually straightforward. However, a number of other lesions of similar histological appearance present a serious challenge in a CNB sample. Such lesions include adenomyoepithelioma, tubular adenoma, juvenile FA, FA with cellular stroma and hamartoma.

The distinction between a benign $\mathrm{PT}$ and a FA with cellular stroma is difficult even after surgical excision of the intact lesion. The distinction is made using some information that may be difficult to assess in the CNB, such as the margin of the lesion, the mitotic rate and the presence of stromal overgrowth. An additional problem is that these lesions commonly show heterogeneity. Furthermore, the criteria used to differentiate FA with cellular stroma from benign PT are poorly defined. The core biopsy diagnosis of FA with cellular stroma is therefore usually managed by surgical excision.

In the CNB study by Lee et al, ${ }^{18}$ eight fibroepithelial lesions with cellular stroma were excised with the result that one was a malignant PT, two were benign and three were borderline PT, and three were FAs, of which one had cellular stroma. In a more recent study from the same group, ${ }^{19} 36 \mathrm{PT}$ had been diagnosed after surgical excision following CNB in 44 patients. Of the latter, PT had been suggested in 32 and a spindle cell lesion in 1 , all of which were excised. The 11 patients in whom an FA was the CNB diagnosis proceeded to surgical excision because they were increasing in size or were large lesions. Review of the 11 core biopsies showed heterogeneity of stromal cellularity. Features that were assessed as helpful in suggesting a PT on core biopsy were:

- Stromal cellularity increased in $50 \%$ or more of the cores when compared with FA.

- Stromal overgrowth; assessed by $\times 10$ field with no epithelium present.

- Fragmentation of the lesion.

- Adipose tissue within the stroma of the lesion.

Jacobs et $a l,{ }^{20}$ studied 29 cases diagnosed as fibroepithelial lesions with cellular stroma on $\mathrm{CNB}$, all of which had undergone surgical excision and follow-up. Using a panel of immunohistochemical markers and analysing a number of pathology features, the authors suggested the following management plan:

- FAs with cellular stroma with markedly increased stromal cellularity are most likely to be PT and should be managed by excision, with attention paid to achieving clear margins.

- Moderately increased stromal cellularity in these lesions, stromal mitoses and elevated Ki67 or topo II predict a high probability of being PT, and excision with clear margins is desirable.

- Moderately increased stromal cellularity, no stromal mitoses and low Ki67 or topo II is consistent with either PT or FA and although these lesions should be excised, attention to margins is less important in the first instance. 
As discussed further below, one option for the management of the last category might be to attempt excision by VACB rather than by open surgery. This treatment technique remains to be fully evaluated by clinical trails. A further option of short-term followup with repeat imaging at 6-12 months could also be considered for this group of patients. The growth of the lesion could then be assessed; rapid growth prompting the decision to surgically excise. Any management decision, however, would need to take into account patient preferences including the anxiety and stress accompanying the uncertainty of a 'wait and see' policy, as well as the convenience or otherwise of proceeding to surgical excision.

\section{Spindle Cell Lesions}

A wide range of spindle cell lesions may be seen in a core biopsy, and most of these will require surgical excision either to confirm the diagnosis or for the further management of the patient. These lesions include metaplastic carcinoma, PT, myofibroblastoma, angiosarcoma, fibromatosis and nodular fasciitis. These lesions will not be further discussed.

\section{Residual or Recurrent Carcinoma after Radiation Therapy}

Core biopsy is often performed to assess microcalcifications seen in the irradiated breast after breast conservation and radiation therapy for invasive carcinoma or DCIS. The calcifications may be seen in areas of fat necrosis with associated hyalinized fibrous scar tissue, or within sutures or other intraoperative material. Microcalcification may also be seen in recurrent/residual DCIS or invasive carcinoma and in benign ducts and TDLUs subjected to irradiation.

The pathology features seen in a breast irradiated for carcinoma resemble those of DCIS, particularly when the DCIS involves the TDLU which are the site of the most pronounced changes after irradiation (Figure 2). The main changes seen in a CNB after irradiation are $^{21}$

- Atrophy of the epithelium of the ducts and the TDLU.

- Cytological atypia of the epithelial cells-this may be patchy and focal even in a single TDLU.

- Prominent myoepithelial cells particularly in the TDLU with associated thickened basement membranes.

- Increased collagen in the breast stroma.

- Atypical stromal fibroblasts.

- Microcalcification within the TDLU and ducts.

Features that may be helpful in performing the diagnosis of DCIS in the irradiated breast include the presence of mitoses and the similarity of the changes to DCIS in the pre-irradiated breast both in
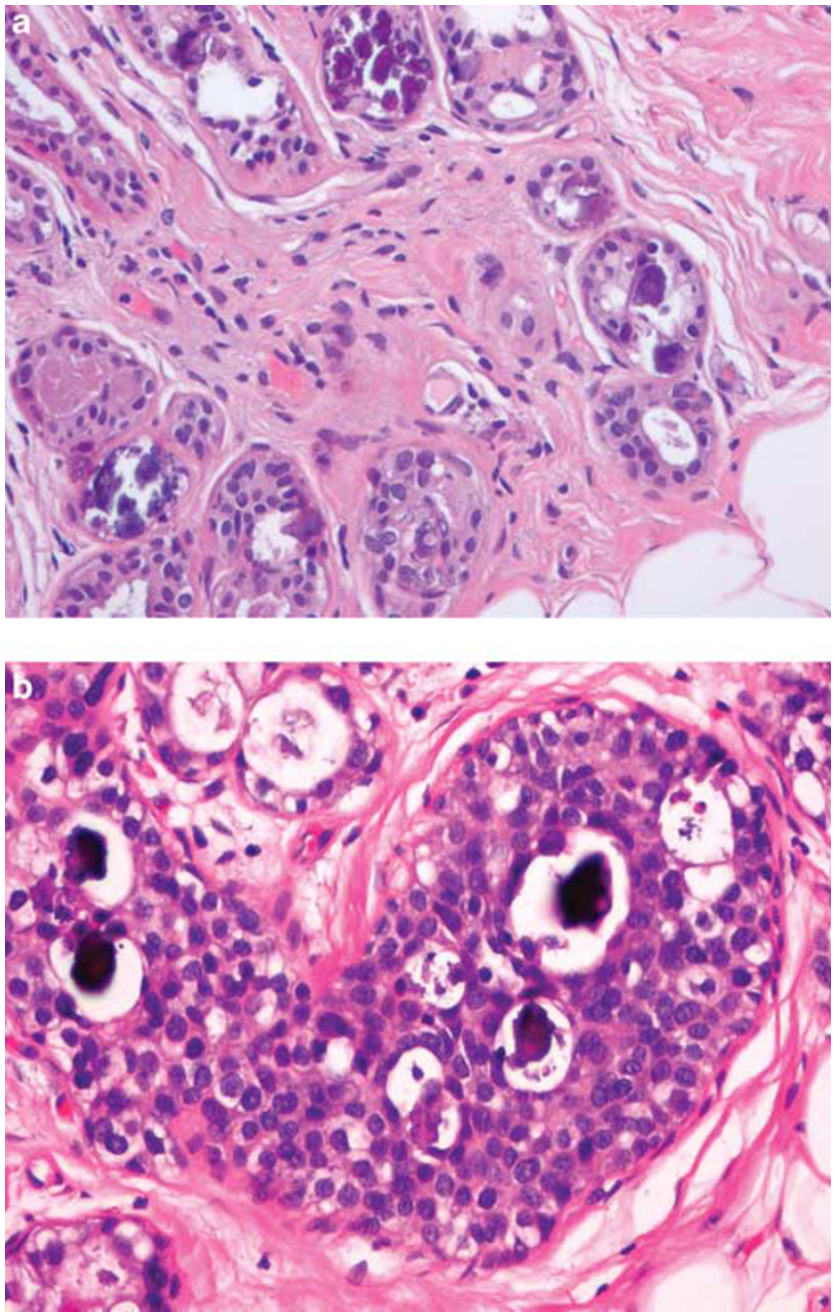

Figure 2 (a) Radiation therapy changes in a terminal duct lobular unit after treatment for DCIS; (b) Recurrent DCIS after breast conserving surgery and radiation therapy for DCIS.

architecture and nuclear grade, as both can remain unchanged in the irradiated breast.

\section{Papillary Lesions}

A spectrum of papillary lesions may be encountered in a CNB sample. These include intraduct papilloma, intraduct papilloma with atypia, intracystic (encapsulated) papillary carcinoma, solid papillary carcinoma and invasive papillary carcinoma. The current policy in most units is to excise all papillary lesions diagnosed on CNB, to allow complete evaluation, but this approach is being challenged. The option of prolonged follow-up with imaging has been suggested as an alternative approach. What is agreed is that all papillary lesions that are palpable or symptomatic should be excised, and any papillary lesion with atypia must also be excised. What is challenged is the policy that a diagnosis of intraduct papilloma on CNB should be followed by surgical excision of the lesion in all cases. The basis of this policy is that intraduct papillary lesions are fre- 
quently heterogeneous and a CNB sample risks missing a more significant area of the lesion.

The diagnosis of intraduct papilloma in the CNB (and the exclusion of intraduct papillary carcinoma) is greatly assisted by good quality H\&E sections and immunohistochemistry. The latter is used primarily to confirm the presence of myoepithelial cells in the lesion (Figure 3). Smooth muscle myosin heavy chain, CD10 and p63 seem to produce the most consistent and specific results. Other features of intraduct papillary carcinoma, but based on excision specimens, have been well described by Kraus and Neubecker. ${ }^{22}$ Intraduct papillomas can have areas of epithelial hyperplasia or atypia equivalent to $\mathrm{ADH}$, and these changes can be difficult to differentiate from intraduct papillary carcinoma. 'Double immunostaining' with p63 and high-molecular-weight cytokeratin has proven to be useful in evaluating these problem cases. ${ }^{23}$

If the diagnosis of intraduct papilloma is made from the CNB, what is the risk of intraduct papillary carcinoma being found on excision? Liberman et $a l^{24}$
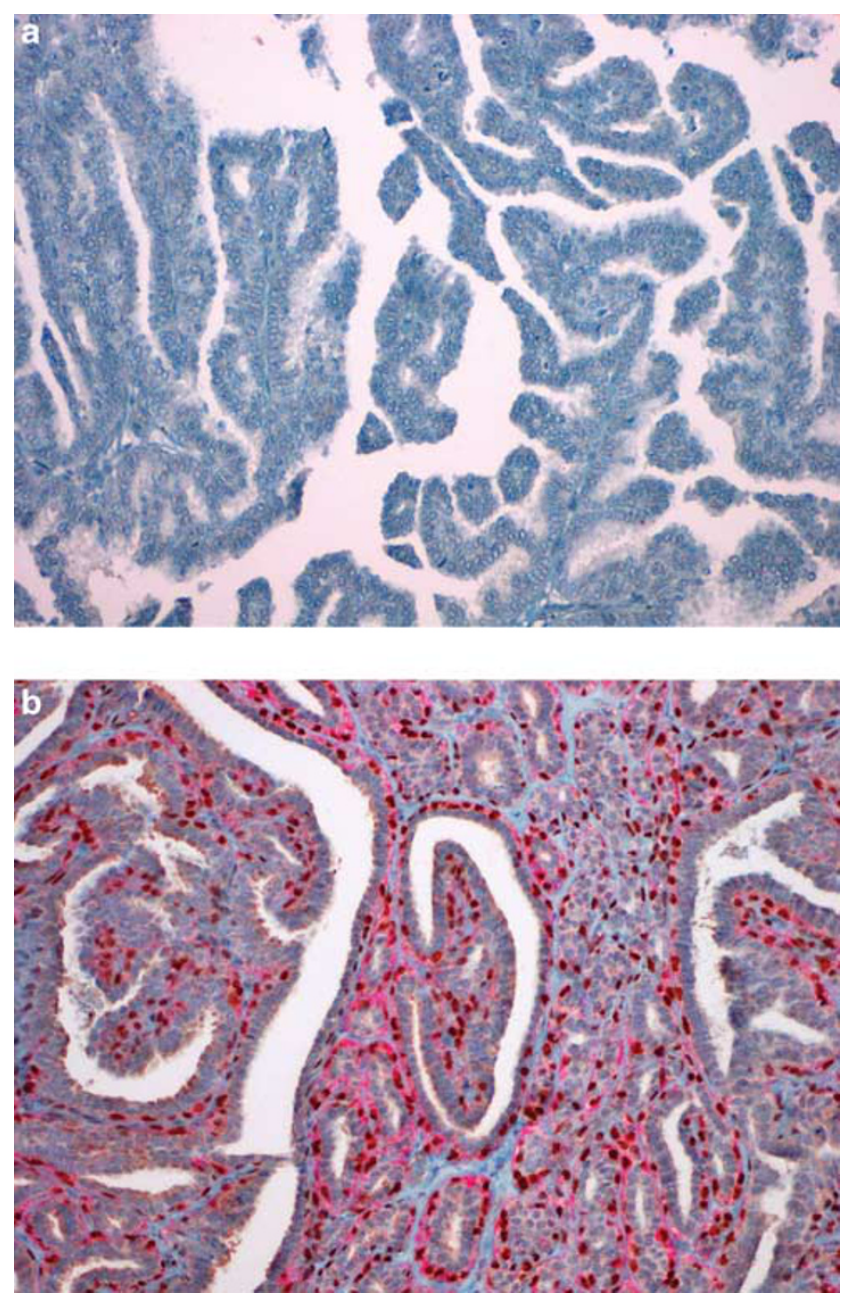

Figure 3 (a) Intracystic papillary carcinoma showing absence of myoepithelial cells by IHC staining (p63); (b) intraduct papilloma showing numerous myoepithelial cells by IHC staining (dual staining p63 + smooth muscle myosin heavy chain). reviewed 35 patients who had a diagnosis of intraduct papilloma on CNB (conventional and VACB). On follow-up, there was a $14 \%$ incidence of carcinoma (four patients with DCIS, one with invasive carcinoma) and in the remaining patients a 'high-risk' lesion was found in 17\% (three ADH, two radial scars and one LCIS). In a literature review, Jacobs et $a l^{25}$ reported an incidence of carcinoma in $0-25 \%$ of patients after a CNB finding of a papillary lesion without atypia. In a series of 38 patients, the same author reported an incidence of intraductal carcinoma in the excision specimen in three cases who had CNB finding of a papilloma without atypia. ${ }^{26}$ Valdes et $a l^{27}$ showed that stereotactic guidance was more effective in obtaining material that accurately predicted malignancy at excision.

Any decision regarding further management of a patient based on CNB findings of an intraduct papilloma is therefore problematic and imaging does not usually offer any further assistance.

Surgical excision of all papillomas has the additional benefit of addressing the, albeit low, risk of carcinoma seen in follow-up studies of patients who have had surgical excision of their papilloma. The risk seems to be mainly in those patients who have multiple papillomas. ${ }^{28}$

A further possible management option may be vacuum-assisted core excision, avoiding surgery. This would have to be subject to careful patient selection, including pathology review of the original core biopsy findings. To date, only a few studies of this procedure are published with limited follow-up available. ${ }^{29-31}$

In summary, there appears to be insufficient evidence to support a general change to the current protocol of surgical excision of intraduct papillomas. The exception could be those small intraduct papillomas with no atypical features (category B2), generously sampled by VACB and showing no residual lesion in post-core imaging. In the future, it can be expected that more studies of vacuumassisted core excision will provide evidence as to whether this is a safe alternative to surgery.

\section{Mucinous Lesions}

Although mucin may be seen as a minor component in invasive carcinomas, pure mucinous carcinoma is rare and is seen more often in older women. ${ }^{32,33}$ Mucinous carcinoma presents as a palpable lump or well-defined mass on mammography. Associated microcalcifications may be seen within the mucin.

The other main source of mucin is a mucocoelelike lesion. In these lesions, mucin is seen within the breast stroma and the origin is from rupture of a duct. The epithelium that lines these ducts can show a range of changes including cuboidal or columnar cells, hyperplasia, atypia and DCIS. ${ }^{34}$ There is often associated microcalcification which may take a variety of forms. Similar to mucinous 
carcinoma, mucocoele-like lesions can present as a well-defined mass or mammographic density with calcifications.

The diagnosis of mucinous carcinoma in a CNB is uncomplicated if neoplastic epithelial cells are seen within the mucin. The diagnosis of a mucocoele-like lesion with a frequently minimal epithelial component is more difficult (Figure 4).
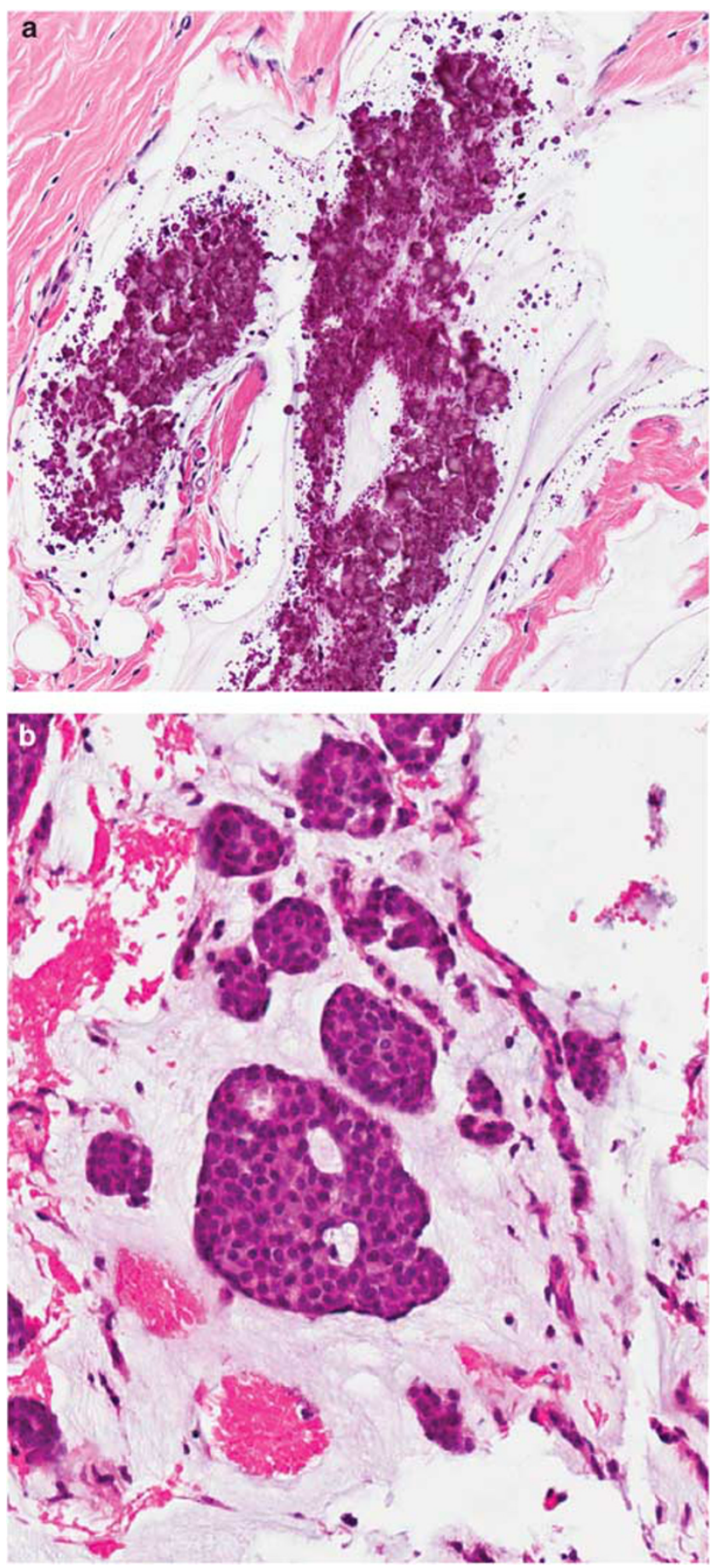

Figure 4 (a) Mucocoele-like lesion with cystic ducts containing mucin and granular microcalcifications but no epithelial cells; (b) mucinous carcinoma with groups of carcinoma cells in mucin pools.
It is reasonable to view mucocoele-like lesions as part of a spectrum from benign through to mucinous carcinoma with a significant risk of underdiagnosis from the limited material present in a CNB. ${ }^{35}$ Excision of mucocoele-like lesions is therefore recommended.

\section{Radial Scar}

Radial scars are composed of a central fibrotic and variably elastotic centre from which an epithelial component extends outwards in radial manner, the whole forming a stellate lesion seen on mammographic imaging. The epithelial component may show florid epithelial hyperplasia, sclerosing adenosis, apocrine change, ADH, DCIS or LN. There may also be papillary change in some of the ducts. Calcification can be seen in association with any of these epithelial changes. Within the central fibrotic core, there are often tubules that closely resemble those seen in invasive carcinoma, especially tubular carcinoma, and these may also be seen at the periphery of the lesion.

The sample of the radial scar seen in a CNB may present significant diagnostic problems. First, there is the need to exclude the diagnosis of invasive carcinoma. This is assisted by the use of myoepithelial cell immunohistochemistry (p63, CD10, smooth muscle myosin heavy chain) to demonstrate a myoepithelial cell component in the small ducts that may be present. Second, there may be DCIS (and/or invasive carcinoma) focally present in any of the ducts at the periphery of the radial scar and this may not be present in the CNB material.

There is some evidence that the greater volume of material sampled by VACB and the absence of atypia predict for a benign diagnosis after surgical excision or follow-up. ${ }^{36}$ In some centres, however, the imaging suggestion of a radial scar is followed by surgical excision without pre-operative CNB.

An added complication in the interpretation of excised radial scars is the post-CNB track with the potential for epithelial displacement simulating invasive carcinoma.

There is some evidence that radial scars are a risk factor for the development of invasive carcinoma in follow-up studies, with the greatest risk being predicted by the increasing size of the lesion and the age of the patient. ${ }^{37}$

It is recommended that radial scars are surgically excised for complete histological assessment. An exception could be argued for those small radial scars thoroughly sampled (excised) in a VACB and showing no atypical features. These patients would require close follow-up with imaging.

\section{Microcalcifications without a Cause?}

Occasionally, microcalcifications are seen in a CNB lying within adipose tissue, the breast stroma or 
a
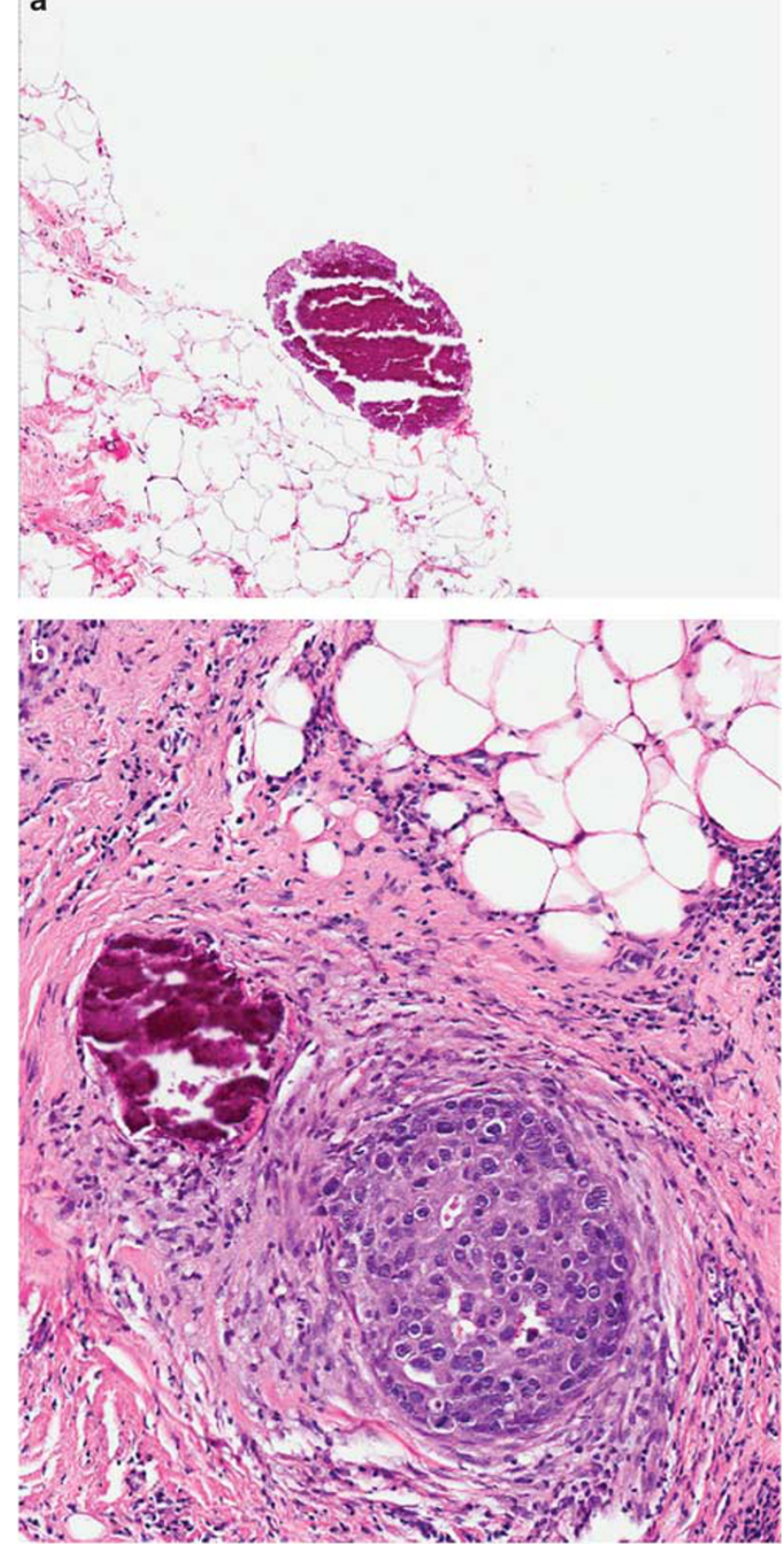

Figure 5 (a) Coarse microcalcification loosely associated with a CNB composed of adipose tissue only; (b) same patient after VACB showing high grade DCIS with comedonecrosis and associated coarse microcalcifications.

loosely adherent to the edge of one of the cores. No apparent cause or associated lesion is seen. In these cases, radiological-pathological correlation is essential to evaluate the possible origin of the calcium. In the case of coarse microcalcifications, there is a high index of suspicion that there may be DCIS with comedonecrosis in breast tissue adjacent to the site of CNB. A related phenomenon is seen in cases of DCIS, in which there is extensive periductal and intraductal fibrosis after necrosis of the epithelium. All that remains as evidence of the DCIS may be

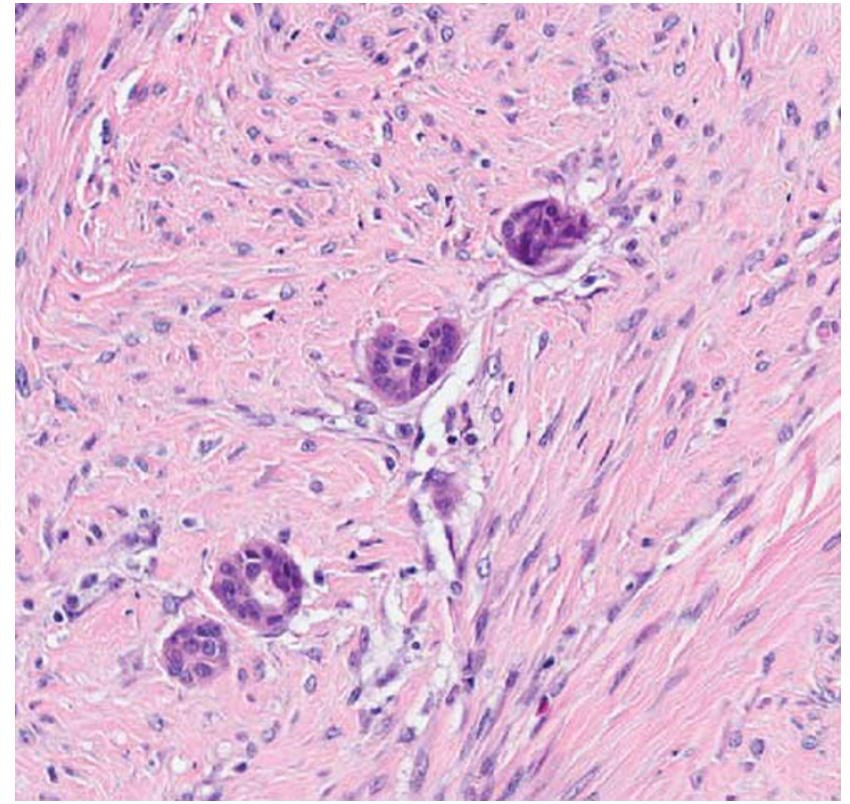

Figure 6 Displaced epithelium derived from an intraduct papilloma seen in the CNB track after surgical excision of the lesion.

occasional epithelial cells and/or microcalcifications within this scar tissue, and scattered periductal lymphocytes. Examining deeper levels and the use of cytokeratin immunohistochemistry to identify any residual DCIS cells may help, but further sampling or excision of the area is frequently required to reach a diagnosis (Figure 5).

\section{Complications and Pathology Findings after CNB}

The incidence of any clinical complications after CNB is low. Significant haematoma, fainting, infection and pneumothorax are reported but are uncommon. Pneumothorax is the most significant complication, and the greatest risk is with posterior lesions in the breast. It is best avoided by taking care not to angle the needle towards the chest wall but parallel to it, and by monitoring the needle position with imaging. Anticoagulants and aspirin may increase the likelihood of haematoma, and prebiopsy clinical history taking is therefore important. The CNB can be deferred until after the anticoagulant dose has been reduced. Compression between samples and/or on completion of the procedure is usually sufficient to prevent significant haematoma.

The changes seen in breast tissue after CNB will vary according to the time interval between the needling procedure and subsequent surgery and also on the number of core samples removed.

The changes include the following:

- Haemorrhage, granulation/fibrous tissue, inflammation in the track of the CNB often with associated histiocytes and haemosiderin-containing macrophages. 
- Disruption and fragmentation of ducts and TDLUs.

- Epidermal inclusion cysts or fragments of epidermis within the breast stroma.

- Displacement of epithelium within the CNB track and in the adjacent breast tissue and occasionally fragments of epithelium and cellular debris within ducts.

- Epithelium within lymphovascular spaces.

Identification of the previous CNB track should be sought in any surgical excision, as evidence that the area of interest has been sampled especially when a clip has not been used to mark the site.

The most problematic change is that of epithelial displacement, which is seen in both benign and malignant lesions, especially lesions with a papillary component (Figure 6). There is some evidence that displaced cells are seen less frequently after VACB than after CNB. ${ }^{38-40}$ The small groups of displaced cells should be stained with a myoepithelial cell marker. However, although the presence of myoepithelial cells is reassuring and confirms the benign nature of the displaced cells, the absence of any myoepithelial cell staining does not imply that the cells are malignant, as they may be derived from an area of a lesion not invested by myoepithelial cells. When the displaced cells are derived from DCIS, the problem of excluding invasive carcinoma is difficult even after comparing them with those seen in the original CNB and the surrounding breast tissue. The same cells can be seen within lymphovascular spaces and within the subcapsular sinus of the sentinel lymph node.

The significance of the displaced cells is not certain and the main concern is that they should not be overdiagnosed as invasive carcinoma, if there is an associated in situ lesion. Similarly, there is uncertainty over the significance of the presence of displaced cells derived from DCIS seen within lymphovascular spaces or the draining lymph nodes. The concept of 'passive transport' of these cells to the lymph node is compelling. However, the alternative view with regard to the cells as representing true metastatic spread from an undiagnosed invasive carcinoma also has its adherents. ${ }^{41}$

\section{Acknowledgement}

I thank Professor Adrienne Morey, St Vincent's Hospital Sydney for the HER2 figures.

\section{Disclosure/conflict of interest}

The author declares no conflict of interest.

\section{References}

1 NHSBSP Guidelines for non-operative diagnostic procedures and reporting in breast cancer screening Publication No. 50 June 2001.
2 Stomper PC, Connolly JL, Meyer JE, et al. Clinically occult ductal carcinoma in situ detected with mammography: analysis of 100 cases with radiologic-pathologic correlation. Radiology 1989;172:235-241.

3 Consensus conference on the classification of ductal carcinoma in situ. The Consensus Conference Committee. Cancer 1997;80:1798-1802.

4 Jackman RJ, Nowels KW, Shepard MJ, et al. Stereotaxic large-core needle biopsy of 450 non palpable breast lesions with surgical correlation in lesions with cancer or atypical hyperplasia. Radiology 1994;193: 91-95.

5 Harris GC, Denley HE, Pinder SE, et al. Correlation of histologic prognostic factors in core biopsies and therapeutic excisions of invasive breast carcinoma. Am J Surg Pathol 2003;27:11-15.

6 Hoda SA, Harigopal M, Harris GC, et al. Expert opinion: what should be included in reports of needle core biopsies of breast? Histopathol 2003;43:87-90.

7 Mcllhenny C, Doughty JC, George WD, et al. Optimum number of core biopsies for accurate assessment of histologic grade in breast cancer. $\mathrm{Br} \mathrm{J}$ Surg 2002;89:84-85.

8 Rhodes A, Jasani B, Barnes D, et al. Reliability of immunohistochemical demonstration of oestrogen receptors in routine practice: interlaboratory variance in the sensitivity of detection and evaluation of scoring systems. J Clin Pathol 2000;53:125-130.

9 Layfield L, Gupta D, Mooney E. Assessment of tissue oestrogen and progesterone receptor levels: a survey of current practice, techniques and quantitation methods. Breast J 2000;6:189-196.

10 Wood B, Junckerstorff R, Sterrett G, et al. A comparison of immunohistochemical staining of oestrogen receptor, progesterone receptor and HER-2 in breast core biopsies and subsequent excisions. Pathology 2007;39:391-395.

11 Jacobs TW, Siziopikou KP, Prioleau JE, et al. Do prognostic marker studies on core needle biopsy specimens of breast carcinoma accurately reflect the marker status of the tumor? Mod Pathol 1998;11: 259-264.

12 Mann G, Fahey V, Feleppa F, et al. Reliance on hormone receptor assays of surgical specimens may compromise outcome in patients with breast cancer. J Clin Oncol 2005;23:5148-5154.

13 Mueller-Holzner E, Fink V, Frede T, et al. Immunohistochemical determination of HER2 expression in breast cancer from core biopsy specimens: a reliable predictor of HER2 status of the whole tumour. Breast Cancer Res Treat 2001;69:13-19.

14 Connor CS, Tawfik AJ, Davis MS, et al. A comparison of prognostic tumor markers obtained on image-guided breast biopsies and final surgical excisions. Am J Surg 2002;184:322-324.

15 Taucher S, Rudas M, Mader RM, et al. Prognostic markers in breast cancer: the reliability of HER2/neu status in core needle biopsy of 325 patients with primary breast cancer. Wien Klin Wochenschr 2004;116:26-31.

16 Burge CN, Chang HR, Apple SK. Do histologic features and results of breast cancer biomarker studies differ between core biopsy and surgical excision specimens? The Breast 2006;2:167-172.

17 Cavaliere A, Sidoni A, Scheibel M, et al. Biopathologic profile of breast cancer core biopsy: is it always a valid method? Cancer Lett 2005;218:117-121. 
18 Lee AHS, Denley HE, Pinder SE, et al. Excision biopsy findings of patients with breast needle core biopsies reported as suspicious of malignancy (B4) or lesion of uncertain malignant potential (B3). Histopathol 2003;42:331-336.

19 Lee AHS, Hodi Z, Ellis IO, et al. Histologic features useful in the distinction of phyllodes tumour and fibroadenoma on needle core biopsy of the breast. Histopathol 2007;51:336-344.

20 Jacobs TW, Chen Y-Y, Guinee DG, et al. Fibroepithelial lesions with cellular stroma on breast core needle biopsy; are there predictors of outcome on surgical excision? Am J Clin Pathol 2005;124:342-354.

21 Moore GH, Schiller JE, Moore GK. Radiation-induced histopathologic changes of the breast. The effects of time. Am J Surg Pathol 2004;28:47-53.

22 Kraus FT, Neubecker RD. The differential diagnosis of papillary tumours of the breast. Cancer 1962;15:444-455.

23 Ichihara S, Fujimoto T, Hashimoto K, et al. Double staining with p63 and high molecular weight cytokeratins distinguishes borderline papillary lesions of the breast. Pathol Int 2007;57:126-132.

24 Liberman L, Tornos C, Huzjan R, et al. Is surgical excision warranted after benign, concordant diagnosis of papilloma at percutaneous breast biopsy? Am J Roentgenol 2006;186:1328-1334.

25 Jacobs TW, Connolly JL, Schnitt SJ. Nonmalignant lesions in breast core needle biopsies: to excise or not to excise? Am J Surg Pathol 2002;26:1095-1110.

26 Jacobs TW, Guinee DG, Holden J, et al. Intraductal papillomas without atypia on breast core needle biopsy (CNB): surgical excision is advisable. Mod Pathol 2005;18(Suppl):37A.

27 Valdes EK, Tartter PI, Genelus-Dominique E, et al. Significance of papillary lesions at percutaneous breast biopsy. Ann Surg Oncol 2006;13:480-482.

28 Carter D. Intraductal papillary tumors of the breast. A study of 76 cases. Cancer 1977;39:1689-1692.

29 Fine RE, Boyd BA, Whitworth PW, et al. Percutaneous removal of benign breast masses using a vacuumassisted hand held device with ultrasound guidance. Am J Surg 2002;184:332-336.
30 Mathew J, Crawford DJ, Lwin M, et al. Ultrasoundguided, vacuum-assisted excision in the diagnosis and treatment of clinically benign breast lesions. Ann R Coll Surg Engl 2007;89:494-496.

31 Vargas HI, Vargas MP, Gonzalez K, et al. Percutaneous excisional biopsy of palpable breast masses under ultrasound visualization. Breast J 2006;12(Suppl): S218-S222.

32 Rosen PP, Lesser ML, Kinne DW. Breast carcinoma at the extremes of age: a comparison of patients younger than 35 years and older than 75 years. J Surg Oncol 1985;28:90-96.

33 Rosen PP, Wang T-Y. Colloid carcinoma of the breast. Analysis of 64 patients with long-term follow-up. Am J Clin Pathol 1980;73:30.

34 Hamele-Bena D, Cranor ML, Rosen PP. Mammary mucocoele-like lesions: benign and malignant. Am J Surg Pathol 1996;20:1081-1085.

35 Weaver MG, Abdul-Karim FW, al-Kaisi N. Mucinous lesions of the breast: a pathological continuum. Pathol Res Pract 1993;189:873-876.

36 Brenner RJ, Jackman RJ, Parker SH, et al. Percutaneous core needle biopsy of radial scars of the breast: when is excision necessary? Am J Roentgenol 2002;179: 1179-1184.

37 Sloane JP, Mayers MM. Carcinoma and atypical hyperplasia in radial scars and complex sclerosing lesions: importance of lesion size and patient age. Histopathol 1993;23:225-231.

38 Diaz LK, Wiley EL, Venta LA. Are malignant cells displaced by large-gauge needle core biopsy of the breast? Am J Roentgenol 1999;173:1303-1313.

39 Boppana S, May M, Hoda S. Does prior fine-needle aspiration cause diagnostic difficulties in histologic evaluation of breast carcinomas? Lab Invest 1994;70:13A.

40 Lee KC, Chan JK, Ho LC. Histologic changes in the breast after fine needle aspiration. Am J Surg Pathol 1994;18:1039-1047.

41 King TA, Ganaraj A, Fey JV, et al. Cytokeratin-positive cells in sentinel lymph nodes in breast cancer are not random events. Experience in patients undergoing prophylactic mastectomy. Cancer 2004;101:926-933. 